\title{
Shear deformation effect in the dynamic analysis of plates stiffened by parallel beams
}

\author{
E. J. Sapountzakis \& V. G. Mokos \\ School of Civil Engineering, National Technical University of Athens, \\ Greece
}

\begin{abstract}
In this paper a general solution for the dynamic analysis of shear deformable stiffened plates subjected to arbitrary loading is presented. According to the proposed model, the stiffening beams are isolated from the plate by sections in the lower outer surface of the plate, taking into account the arising tractions in all directions at the fictitious interfaces. These tractions are integrated with respect to each half of the interface width resulting two interface lines, along which the loading of the beams as well as the additional loading of the plate is defined. Their unknown distribution is established by applying continuity conditions in all directions at the interfaces. The utilization of two interface lines for each beam enables the nonuniform distribution of the interface transverse shear forces and the nonuniform torsional response of the beams to be taken into account. The analysis of both the plate and the beams is accomplished on their deformed shape taking into account second-order effects. The analysis of the plate is based on Reissner's theory, while the analysis of the beams is based on Timoshenko's beam theory. Six boundary value problems are formulated and solved using the Analog Equation Method (AEM), a BEM based method.
\end{abstract}

Keywords: ribbed plate, Reissner's theory, Timoshenko's beam theory, dynamic analysis, boundary element method.

\section{Introduction}

Structural plate systems stiffened by beams are widely used in buildings, bridges, ships, aircrafts and machines. Moreover, for cases wherein the plate or the beams are not very "thin" or the stiffeners are closely spaced, the error incurred from the ignorance of the effect of shear deformation may be substantial, while the 
accuracy of a classical analysis decreases and the truthfulness of the results is lost with growing plate or beam thickness. The extensive use of the aforementioned plate structures necessitates a rigorous analysis. The behaviour of stiffened plates has been widely studied employing the finite element method $[1,2]$, the boundary element method $[3,4,5]$ or a combination of these methods [6]. In all these approximations the solution of the bending problem of stiffened plates is not general since either the analysis of the plate and the beams is performed on the undeformed shape ignoring second-order effects or the shear longitudinal or transverse forces at the interfaces have been neglected or the torsional and warping behaviour of the stiffening beams has been ignored excluding in this way the placement of an eccentric stiffener. Only Sapountzakis and Mokos in [7] presented a general solution for the dynamic analysis of plates stiffened by parallel beams taking into account tractions in all directions at the fictitious plate - beams interfaces and enabling the nonuniform distribution of the interface transverse shear forces and the nonuniform torsional response of the beams to be taken into account. In all of the aforementioned research efforts shear deformation effect has been ignored. Contrary to the extended literature concerning the analysis of plates reinforced with beams ignoring shear deformation effect, relatively little work has been done only on the static analysis of shear deformable stiffened plates. The FEM has been employed using shear deformation theory [8,9] while the BEM has been used by Wen et al. [10] by coupling the shear deformable plate formulation and the two-dimensional plane stress elasticity. Also in these latter research efforts the solution of the bending problem of stiffened plates is not general for the same reasons mentioned in the previous paragraph. To the authors' knowledge shear deformation effect has not yet been taken into account in the dynamic analysis of plates reinforced with beams. In this paper a general solution for the dynamic analysis of plates stiffened by arbitrarily placed parallel beams of arbitrary doubly symmetric cross section subjected to arbitrary dynamic loading is presented taking into account shear deformation effect in both the plate and the beams. The employed structural model is the one presented by Sapountzakis and Mokos in [7], in which the nonuniform distribution of the interface transverse shear forces and the nonuniform torsional response of the beams are taken into account. According to this model, the stiffening beams are isolated from the plate by sections in the lower outer surface of the plate, taking into account the arising tractions in all directions at the fictitious interfaces. These tractions are integrated with respect to each half of the interface width resulting two interface lines, along which the loading of the beams as well as the additional loading of the plate is defined. The unknown distribution of the aforementioned integrated tractions is established by applying continuity conditions in all directions at the two interface lines. The analysis of both the plate and the beams is accomplished on their deformed shape taking into account second-order effects. The method of analysis is based on the capability to establish a flexibility matrix with respect to a set of nodal mass points, while a lumped mass matrix is constructed from the tributary mass areas to these mass points. The analysis of the plate is based on Reissner's theory, which may be considered as the standard thick plate theory with which all others 
are compared, while the analysis of the beams is performed employing the linearized second order theory taking into account shear deformation effect. Six boundary value problems are formulated and solved using the Analog Equation Method (AEM) [11], a BEM based method.

\section{Statement of the problem}

Consider a plate of homogeneous, isotropic and linearly elastic material with modulus of elasticity $E$, shear modulus $\mathrm{G}$ and Poisson ratio $\mu$, having constant thickness $h_{p}$ and occupying the two-dimensional multiply connected region $\Omega$ of the $x, y$ plane bounded by the piecewise smooth $\mathrm{K}+1$ curves $\Gamma_{0}, \Gamma_{1}, \ldots, \Gamma_{K-1}, \Gamma_{K}$, as shown in Fig.1. The plate is stiffened by a set of $i=1,2, \ldots, I$ arbitrarily placed parallel beams of arbitrary doubly symmetric cross section of homogeneous, isotropic and linearly elastic material with modulus of elasticity $E_{b}^{i}$, shear modulus $G_{b}^{i}$ and Poisson ratio $\mu_{b}^{i}$, which may have either internal or boundary point supports. For the sake of convenience the $x$ axis is taken parallel to the beams. The stiffened plate is subjected to the lateral load $g=g(\mathbf{x}, t), \mathbf{x}:\{x, y\}, t \geq 0$. For the analysis of the aforementioned problem a global coordinate system $O x y$ for the analysis of the plate and local coordinate ones $O^{i} x^{i} y^{i}$ corresponding to the centroid axes of each beam are employed as shown in Fig.1.

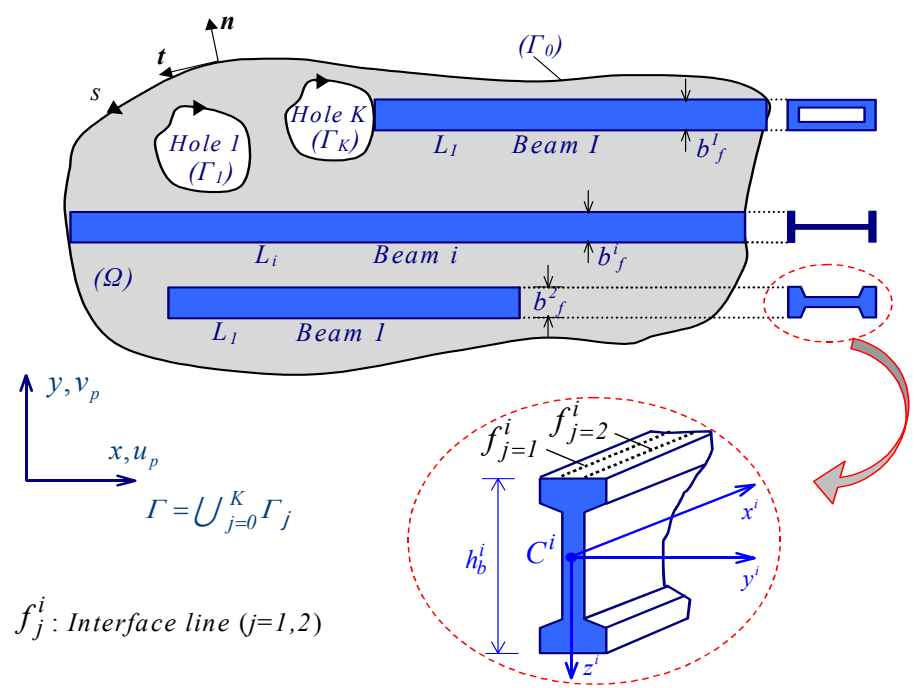

Figure 1: $\quad$ Two-dimensional region $\Omega$ occupied by the plate. 
According to the proposed model, the stiffening beams are isolated from the plate by sections in its lower outer surface, taking into account the arising tractions at the fictitious interfaces. Integration of these tractions along each half of the width of the $\mathrm{i}$-th beam results in line forces per unit length in all directions in two interface lines, which are denoted by $q_{x j}^{i}, q_{y j}^{i}$ and $q_{z j}^{i} \quad(j=1,2)$ encountering in this way the nonuniform distribution of the interface transverse shear forces $q_{y}^{i}$. The aforementioned integrated tractions result in the loading of the i-th beam as well as the additional loading of the plate. Their distribution is unknown and can be established by imposing displacement continuity conditions in all directions along the two interface lines, enabling in this way the nonuniform torsional response of the beams to be taken into account.

\subsection{Initial boundary value problems}

On the base of the above considerations the response of the plate and the beams may be described by the following initial boundary value problems.

\subsubsection{For the plate}

The plate undergoes transverse deflection and inplane deformation. Thus for the transverse deflection, according to Reissner's theory and employing the linearized second order theory, the equation of motion can be written as

$$
\begin{aligned}
& D \nabla^{4} w_{p}+\rho_{p} \ddot{w}_{p}+c_{p} \dot{w}_{p}-\left(N_{x} \frac{\partial^{2} w_{p}}{\partial x^{2}}+2 N_{x y} \frac{\partial^{2} w_{p}}{\partial x \partial y}+N_{y} \frac{\partial^{2} w_{p}}{\partial y^{2}}\right)=g-\frac{h_{p}^{2}}{10} \frac{2-\mu}{1-\mu} \nabla^{2} g- \\
& -\sum_{i=1}^{I}\left(\sum_{j=1}^{2}\left(q_{z j}^{i}-\frac{h_{p}^{2}}{10} \frac{2-\mu}{1-\mu} \nabla^{2} q_{z j}^{i}-\frac{\partial m_{p x j}^{i}}{\partial y}+\frac{\partial m_{p y j}^{i}}{\partial x}-q_{x j}^{i} \frac{\partial w_{p j}^{i}}{\partial x}-q_{y j}^{i} \frac{\partial w_{p j}^{i}}{\partial y}\right) \delta_{j}^{i}\left(y-y_{j}\right)\right) \\
& \text { in } \Omega \text { (1) } \\
& \alpha_{p 1} w_{p}+\alpha_{p 2} Q_{p n}=\alpha_{p 3} \\
& \beta_{p 1} \phi_{p n}+\beta_{p 2} M_{p n}=\beta_{p 3} \quad \text { on } \Gamma \\
& \gamma_{p 1} \phi_{p t}+\gamma_{p 2} M_{p n t}=\gamma_{p 3} \\
& w_{p}(\mathbf{x}, 0)=w_{p 0}(\mathbf{x}) \quad \dot{w}_{p}(\mathbf{x}, 0)=\bar{w}_{p 0}(\mathbf{x})
\end{aligned}
$$

where $D=E h_{p}^{3} / 12\left(1-v^{2}\right) \quad$ is its flexural rigidity; $N_{x}=N_{x}(\mathbf{x}, t)$, $N_{y}=N_{y}(\mathbf{x}, t), N_{x y}=N_{x y}(\mathbf{x}, t)$ are the membrane forces per unit length of the plate cross section; $\rho_{p}=\rho h_{p}$ is the surface mass density of the plate with $\rho$ being the volume mass density; $c_{p}$ is the plate flexural damping constant; $\delta\left(y-y_{i}\right)$ is the Dirac's delta function in the y direction. Also, $a_{p l}, \beta_{p l}, \gamma_{p l}$ $(l=1,2,3)$ are given functions specified on the boundary $\Gamma ; w_{p 0}(\mathbf{x}), \bar{w}_{p 0}(\mathbf{x})$ 
are the initial deflection and the initial velocity of the points of the middle surface of the plate; $Q_{p n}, M_{p n}, M_{p n t}$ are the shear force, the bending moment and the twisting moment along the boundary, respectively and $\phi_{p n}, \phi_{p t}$ are the average rotations of the plate with respect to the axes $t, n$, respectively.

Since linearized plate bending theory is considered, the components of the membrane forces $N_{x}, N_{y}, N_{x y}$ are given as

$$
\begin{gathered}
N_{x}=C\left(\frac{\partial u_{p}}{\partial x}+\mu \frac{\partial v_{p}}{\partial y}\right) \quad N_{y}=C\left(\mu \frac{\partial u_{p}}{\partial x}+\frac{\partial v_{p}}{\partial y}\right) \\
N_{x y}=C \frac{1-\mu}{2}\left(\frac{\partial u_{p}}{\partial y}+\frac{\partial v_{p}}{\partial x}\right)
\end{gathered}
$$

where $C=E h_{p} /\left(1-\mu^{2}\right) ; u_{p}=u_{p}(x, y), v_{p}=v_{p}(x, y)$ are the displacement components of the middle surface of the plate arising from the line body forces $q_{x j}^{i}, q_{y j}^{i}(\mathrm{i}=1,2, \ldots \mathrm{I}),(\mathrm{j}=1,2)$. These displacement components are established by solving independently the plane stress problem, which is described by the following quasi-static (inplane inertia forces are ignored) boundary value problem (Navier's equations of equilibrium)

$$
\begin{gathered}
\nabla^{2} u_{p}+\frac{1+\mu}{1-\mu} \frac{\partial}{\partial x}\left[\frac{\partial u_{p}}{\partial x}+\frac{\partial v_{p}}{\partial y}\right]-\frac{1}{G h_{p}} \sum_{i=1}^{I}\left(\sum_{j=1}^{2} q_{x j}^{i} \delta_{j}^{i}\left(y-y_{i}\right)\right)=0 \\
\nabla^{2} v_{p}+\frac{1+\mu}{1-\mu} \frac{\partial}{\partial y}\left[\frac{\partial u_{p}}{\partial x}+\frac{\partial v_{p}}{\partial y}\right]-\frac{1}{G h_{p}} \sum_{i=1}^{I}\left(\sum_{j=1}^{2} q_{y j}^{i} \delta_{j}^{i}\left(y-y_{i}\right)\right)=0 \quad \text { in } \Omega \\
\delta_{p 1} u_{p n}+\delta_{p 2} N_{n}=\delta_{p 3} \quad \varepsilon_{p 1} u_{p t}+\varepsilon_{p 2} N_{t}=\varepsilon_{p 3} \text { on } \Gamma
\end{gathered}
$$

in which $N_{n}, N_{t}$ and $u_{p n}, u_{p t}$ are the boundary membrane forces and displacements in the normal and tangential directions to the boundary, respectively; $\delta_{p l}, \varepsilon_{p l}(l=1,2,3)$ are functions specified on the boundary $\Gamma$.

\subsubsection{For each (i-th) beam}

Each beam undergoes transverse deflection with respect to $z^{i}$ and $y^{i}$ axes, axial deformation along $x^{i}$ axis and nonuniform angle of twist along $x^{i}$ axis.

Thus, for the transverse deflection with respect to $z^{i}$ axis the equation of motion employing the linearized second order theory and taking into account shear deformation effect can be written as 


$$
\begin{gathered}
E_{b}^{i} I_{y}^{i}\left(1+\frac{N_{b}^{i}}{G_{b}^{i} A_{z}^{i}}\right) \frac{\partial^{4} w_{b}^{i}}{\partial x^{i 4}}+\rho_{b} \ddot{w}_{b}^{i}+c_{b}^{i} \dot{w}_{b}^{i}=\sum_{j=1}^{2}\left(q_{z j}^{i}-q_{x j}^{i} \frac{\partial w_{b}^{i}}{\partial x^{i}}+N_{b j}^{i} \frac{\partial^{2} w_{b}^{i}}{\partial x^{i 2}}-\frac{\partial m_{b y j}^{i}}{\partial x^{i}}\right) \\
-\frac{E_{b}^{i} I_{y}^{i}}{G_{b}^{i} A_{z}^{i}} \sum_{j=1}^{2}\left(\frac{\partial^{2} q_{z j}^{i}}{\partial x^{i 2}}-3 q_{x j}^{i} \frac{\partial^{3} w_{b}^{i}}{\partial x^{i 3}}-3 \frac{\partial q_{x j}^{i}}{\partial x^{i}} \frac{\partial^{2} w_{b}^{i}}{\partial x^{i 2}}-\frac{\partial^{2} q_{x j}^{i}}{\partial x^{i 2}} \frac{\partial w_{b}^{i}}{\partial x^{i}}\right) \\
\text { in } L^{i}, \mathrm{i}=1,2, \ldots, I \quad \text { at the beam ends } x^{i}=0, L^{i} \\
a_{1}^{z i} w_{b}^{i}+a_{2}^{z i} R_{b z}^{i}=a_{3}^{z i} \quad \beta_{1}^{z i} \theta_{b y}^{i}+\beta_{2}^{z i} M_{b y}^{i}=\beta_{3}^{z i} \quad \text { (9a,b) } \\
w_{b}^{i}\left(x^{i}, 0\right)=w_{b 0}^{i}\left(x^{i}\right) \quad \dot{w}_{b}^{i}\left(x^{i}, 0\right)=\bar{w}_{b 0}^{i}\left(x^{i}\right) \quad(10 \mathrm{a}, \mathrm{b})
\end{gathered}
$$

where $\theta_{b y}^{i}, R_{b z}^{i}, M_{b y}^{i}$ are the slope, the reaction and the bending moment at the i-th beam ends, respectively, while $G_{b}^{i} A_{z}^{i}$ is the shear rigidity according to Timoshenko's beam theory. Similarly, the $v_{b}^{i}=v_{b}^{i}\left(x^{i}\right)$ transverse deflection with respect to $y^{i}$ axis must satisfy the following quasi-static (transverse inertia forces with respect to $y^{i}$ axis are ignored) boundary value problem

$$
\begin{gathered}
E_{b}^{i} I_{z}^{i}\left(1+\frac{N_{b}^{i}}{G_{b}^{i} A_{y}^{i}}\right) \frac{\partial^{4} v_{b}^{i}}{\partial x^{i 4}}=\sum_{j=1}^{2}\left(q_{y j}^{i}-q_{x j}^{i} \frac{\partial v_{b}^{i}}{\partial x^{i}}+N_{b j}^{i} \frac{\partial^{2} v_{b}^{i}}{\partial x^{i 2}}-\frac{\partial m_{b z j}^{i}}{\partial x^{i}}\right) \\
-\frac{E_{b}^{i} I_{z}^{i}}{G_{b}^{i} A_{y}^{i}} \sum_{j=1}^{2}\left(\frac{\partial^{2} q_{y j}^{i}}{\partial x^{i 2}}-3 q_{x j}^{i} \frac{\partial^{3} v_{b}^{i}}{\partial x^{i 3}}-3 \frac{\partial q_{x j}^{i}}{\partial x^{i}} \frac{\partial^{2} v_{b}^{i}}{\partial x^{i 2}}-\frac{\partial^{2} q_{x j}^{i}}{\partial x^{i 2}} \frac{\partial v_{b}^{i}}{\partial x^{i}}\right) \\
\operatorname{in} L^{i}, \mathrm{i}=1,2, \ldots, I \quad \text { at the beam ends } x^{i}=0, L^{i}
\end{gathered}
$$

The axial deformation $u_{b}^{i}$ of the beam is described by solving the following quasi-static (axial inertia forces are neglected) boundary value problem

$$
\begin{gathered}
E_{b}^{i} A_{b}^{i} \frac{\partial^{2} u_{b}^{i}}{\partial x^{i 2}}=-\sum_{j=1}^{2} q_{x j}^{i} \quad \text { in } L^{i}, \mathrm{i}=1,2, \ldots, I \\
\gamma_{1}^{x i} u_{b}^{i}+\gamma_{2}^{x i} N_{b}^{i}=\gamma_{3}^{x i} \text { at the beam ends } x^{i}=0, L^{i}
\end{gathered}
$$

Finally, the nonuniform angle of twist $\theta_{b x}^{i}=\theta_{b x}^{i}\left(x^{i}\right)$ with respect to $x^{i}$ shear centre axis has to satisfy the following quasi-static (torsional and warping inertia moments are ignored) boundary value problem

$$
E_{b}^{i} I_{b w}^{i} \frac{\partial^{4} \theta_{b x}^{i}}{\partial x^{i 4}}-G_{b}^{i} I_{b x}^{i} \frac{\partial^{2} \theta_{b x}^{i}}{\partial x^{i 2}}=\sum_{j=1}^{2} m_{b x j}^{i} \quad \text { in } L^{i}, \mathrm{i}=1,2, \ldots, I
$$




$$
a_{1}^{x i} \theta_{b x}^{i}+a_{2}^{x i} M_{b x}^{i}=a_{3}^{x i} \quad \beta_{1}^{x i} \frac{\partial \theta_{b x}^{i}}{\partial x^{i}}+\beta_{2}^{x i} M_{b w}^{i}=\beta_{3}^{x i} \quad x_{i}=0, L_{i}
$$

\subsection{Displacement continuity conditions}

The displacement continuity conditions in the directions of $z^{i}, x^{i}$ and $y^{i}$ local axes along the two interface lines of each (i-th) plate - beam interface can be expressed as

$$
\begin{array}{cc}
w_{p 1}^{i}-w_{b}^{i}=-\frac{b_{f}^{i}}{4} \theta_{b x}^{i} & w_{p 2}^{i}-w_{b}^{i}=\frac{b_{f}^{i}}{4} \theta_{b x}^{i} \\
u_{p 1}^{i}-u_{b}^{i}=\frac{h_{p}}{2} \frac{\partial w_{p 1}^{i}}{\partial x}+\frac{h_{b}^{i}}{2} \frac{\partial w_{b}^{i}}{\partial x^{i}}+\frac{b_{f}^{i}}{4} \frac{\partial v_{b}^{i}}{\partial x^{i}}+\left(\phi_{S}^{i P}\right)_{f 1} \frac{\partial \theta_{b x}^{i}}{\partial x^{i}} \\
u_{p 2}^{i}-u_{b}^{i}=\frac{h_{p}}{2} \frac{\partial w_{p 2}^{i}}{\partial x}+\frac{h_{b}^{i}}{2} \frac{\partial w_{b}^{i}}{\partial x^{i}}-\frac{b_{f}^{i}}{4} \frac{\partial v_{b}^{i}}{\partial x^{i}}+\left(\phi_{S}^{i P}\right)_{f 2} \frac{\partial \theta_{b x}^{i}}{\partial x^{i}} \\
v_{p 1}^{i}-v_{b}^{i}=\frac{h_{p}}{2} \frac{\partial w_{p 1}^{i}}{\partial y}+\frac{h_{b}^{i}}{2} \theta_{b x}^{i} & v_{p 2}^{i}-v_{b}^{i}=\frac{h_{p}}{2} \frac{\partial w_{p 2}^{i}}{\partial y}+\frac{h_{b}^{i}}{2} \theta_{b x}^{i}
\end{array}
$$

where $\left(\phi_{S}^{i P}\right)_{f j}$ is the value of the primary warping function of the beam cross section at the point of the $\mathrm{j}$-th interface line of the $\mathrm{i}$-th plate - beam interface $f$.

\section{Solution procedure}

The numerical solution of the aforementioned problem is achieved employing the method presented by Katsikadelis and Kandilas [12]. According to this method the domain $\Omega$ occupied by the plate is discretized by establishing a system of $M$ nodal points on it, corresponding to $M$ mass cells, to which masses are assigned according to the lumped mass assumption. Subsequently, the stiffness matrix, the damping matrix as well as the load vector with respect to these nodal points are established employing the Analog Equation Method (AEM) [11], a BEM based method. This procedure leads to the typical equation of motion for the stiffened plate

$$
[m]\{\ddot{w}\}+[c]\{\dot{w}\}+[k]\{w\}=\{g\}
$$

\section{Numerical example}

A concrete $C 20 / 25$ rectangular plate with dimensions $18.0 \times 9.0 \mathrm{~m}$ subjected to a uniformly distributed trapezoidal load $g(t)$ and stiffened by two concrete $C 20 / 25$ rectangular beams of $1.0 \mathrm{~m}$ width placed at its free sides (Figure 2) has 
been studied (damping ratio $\xi=\xi_{b}^{i}=0$ ). The plate is clamped along its small edges, while the other two edges are free. In Table 1 the first five eigenperiods of the free vibrating stiffened plate and in Figure 3 the time history of the deflection $w_{p}(t)$ at points $\mathrm{A}, \mathrm{B}$ of the stiffened plate are presented. From these table and figure the significant influence of the inplane forces and the effect of shear deformation are noteworthy.

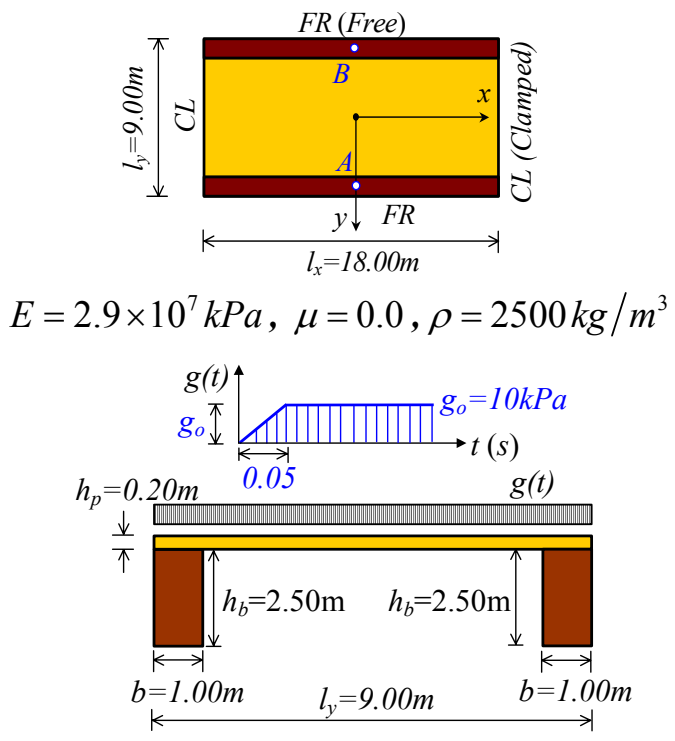

Figure 2: $\quad$ Plan view and section of the stiffened plate of Example 1.

Table 1: $\quad$ Eigenperiods $T_{i}(s)$ of the stiffened plate of Example 1.

\begin{tabular}{|c|c|c|c|c|}
\hline \multirow{2}{*}{$i$} & \multicolumn{2}{|c|}{ Including Inplane Forces } & \multicolumn{2}{c|}{ Ignoring Inplane Forces } \\
\cline { 2 - 5 } & $\begin{array}{c}\text { With Shear } \\
\text { deformation } \\
\text { (Present study) }\end{array}$ & $\begin{array}{c}\text { Without } \\
\text { Shear } \\
\text { deformation }\end{array}$ & $\begin{array}{c}\text { With Shear } \\
\text { deformation }\end{array}$ & $\begin{array}{c}\text { Without Shear } \\
\text { deformation }\end{array}$ \\
\hline 1 & 0.08704 & 0.08668 & 0.09228 & 0.09042 \\
\hline 2 & 0.06701 & 0.06695 & 0.06753 & 0.06738 \\
\hline 3 & 0.05010 & 0.05008 & 0.06399 & 0.05742 \\
\hline 4 & 0.04573 & 0.04356 & 0.05801 & 0.05297 \\
\hline 5 & 0.04268 & 0.04058 & 0.05037 & 0.05005 \\
\hline
\end{tabular}




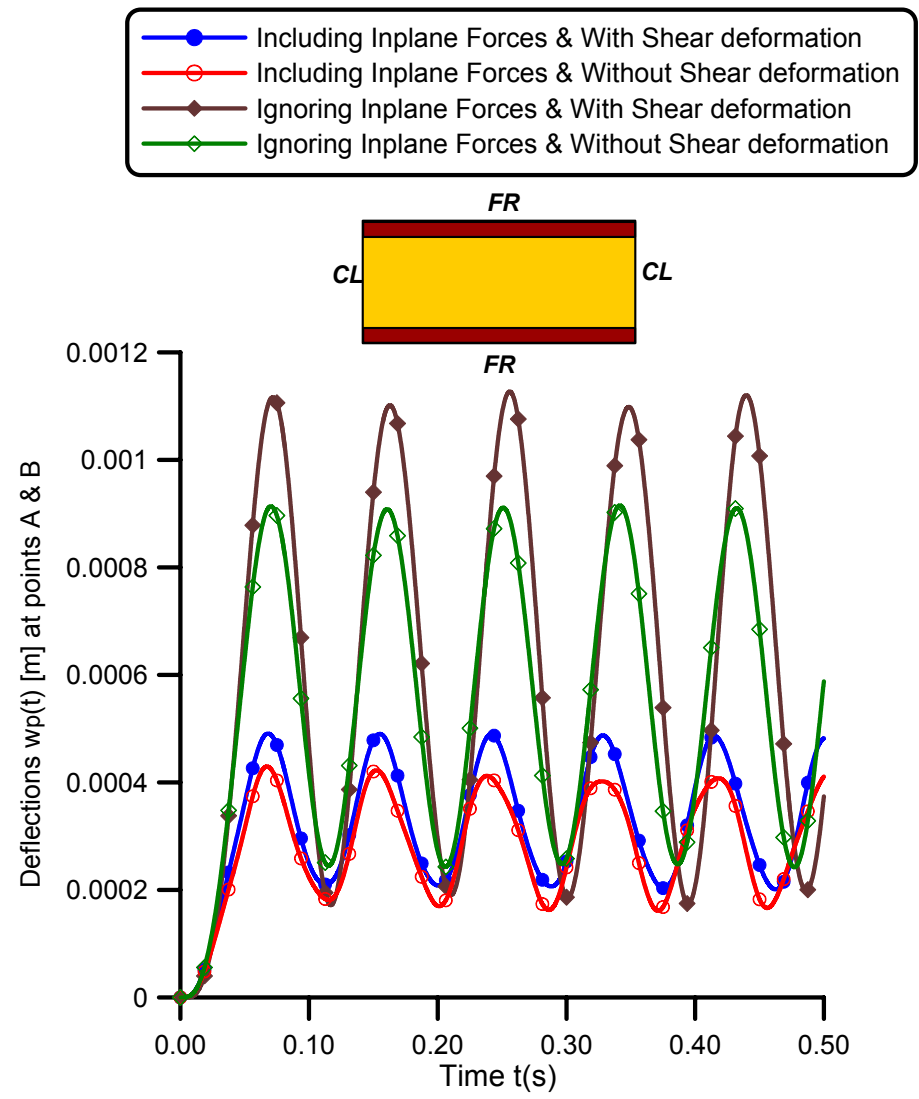

Figure 3: $\quad$ Time history of the deflection $w_{p}(t)$ at points $\mathrm{A}, \mathrm{B}$.

\section{Concluding remarks}

The influence of the inplane forces and the shear deformation effect to the deflections is remarkable and should not be neglected.

\section{References}

[1] Palani GS, Iyer NR, Apa Rao TVSR, An Efficient Finite Element Model for Static and Vibration Analysis of Eccentrically Stiffened Plates/Shells, Computers and Structures, pp.651-661, 1992.

[2] Peng-Cheng S., Dade H., Zongmu W., Static Vibration and Stability analysis of Stiffened Plates Using B Spline Functions, Computers and Structures, pp.73-78, 1993.

[3] Sapountzakis EJ, Katsikadelis JT., Analysis of Plates Reinforced with Beams, Computational Mechanics, 26, pp.66-74, 2000. 
[4] Tanaka M, Matsumoto T, Oida S., A Boundary Element Method Applied to the Elastostatic Bending Problem of Beam-Stiffened Plates, Engineering Analysis with Boundary Elements, 24, 751-758, 2000.

[5] Fernandes GR, Venturini WS., Building Floor Analysis by the Boundary Element Method, Computational Mechanics, 35, pp.277-291, 2005.

[6] Ng SF, Cheung MS, Xu T., A Combined Boundary Element and Finite Element Solution of Slab and Slab-on-Girder Bridges, Computers and Structures, 37, pp.1069-1075, 1990.

[7] Sapountzakis E.J. and Mokos V.G., An Improved Model for the Dynamic Analysis of Plates Stiffened by Parallel Beams, Engineering Structures, 30, pp.1720-1733, 2008.

[8] Biswal KC, Ghosh AK, Finite element analysis for stiffened laminated place using higher order shear deformation theory, Computers and Structures, 53(1), pp.161-71, 1994.

[9] Deb A, Booton M, Finite element models for stiffened plates under transverse loading, Computers and Structures, 28(3), pp.361-372, 1988.

[10] Wen PH, Aliabadi MH, Young A., Boundary element analysis of shear deformable stiffened plates, Engineering Analysis with Boundary Elements, 26, pp.511-520, 2002.

[11] Katsikadelis JT., The Analog Equation Method. A Boundary - only Integral Equation Method for Nonlinear Static and Dynamic Problems in General Bodies, Theoretical and Applied Mechanics, 27, pp.13-38, 2002.

[12] Katsikadelis, J. T. and Kandilas, C.B., A Flexibility Matrix Solution of the Vibration Problem of Plates Based on the Boundary Element Method, Acta Mechanica, 83, pp.51-60, 1990. 